\title{
LES RESSOURCES EN EAU AU CAP BON: DES CONFLITS SOCIO-ENVIRONNEMENTAUX AUX VULNÉRABILITÉS ÉCOSYSTÉMIQUES
}

\author{
Saied SAOUSSEN \\ Lahmar KARIM
}

\begin{abstract}
Tunisia and, in particular, the eastern facade of Cap Bon has considered the integrated management of water and its resources as a challenge and an issue of great importance for human development and the sustainable management of its landscapes. This region has an essentially agricultural vocation but also has a strong tourist attraction thanks to its natural and cultural potential, the current economic context of the coastal towns of Cap Bon induces a rapid development in the demand for water. There is already a competition for water exploitation between the agricultural and tourist sectors. The demand for water is then brought to meet the needs of the tourist industry and those of the agricultural activity, without forgetting the industrial and domestic sector. Although agriculture is the main activity for the majority of the population, agricultural intensification threatens the sustainability of the water resource, as a means of production, both in terms of quality and in terms of quantity. and the threat as well, as natural wealth. We must therefore think about redefining the place of water through the differential management of water resources by considering them not only as a vital and economic resource but also as an environmental and landscape component of great importance. To do this and based on the documentary research and the various surveys carried out in the study area, this article aims to present the different water sources in Cap Bon, to focus on the multiple conflicts of use by different sectors (agriculture, industry, domestic use, etc.) and subsequently to explore what new forms of governance in the hydraulic field can be. The main objective is to plan the use, protection, conservation and sustainable and rational management of water resources according to the needs and priorities of communities, within the framework of national economic and environmental development policies.
\end{abstract}

Keywords: water resources; Cap Bon; conflicts of use; landscape management; sustainable development

DOI: 10.53373 / REDS.2021.52.3.032

\section{Introduction}

Partout dans le monde et plus particulièrement en régions méditerranéennes, la gestion de l'eau est devenue ces dernières années un sujet de préoccupation de forte importance. Cette ressource indispensable se trouve mal répartie (Mutin, 2009). Cette inégalité de distribution et d'exploitation entre les différents secteurs économiques, est en relation avec les conditions climatiques (Riviere-Honegger,

* Enseignante- chercheur à l'Institut Supérieur Agronomique de Chott-Mariem, Tunisie.

** Enseignant- chercheur à l'Université de Monastir, Tunisie., e-mail Lahmar_karim@yahoo.fr. 
2008) qui incitent à l'essor de l'agriculture irriguée et au développement du secteur touristique et industriel tel est le cas de la Tunisie et plus particulièrement la région de la presqu'ile du Cap Bon. En effet, les villes littorales du Cap Bon sont caractérisées par leurs activités agricoles intenses et leurs particularités paysagères, climatiques et culturelles qui attirent de vastes activités touristiques et industrielles. Ces secteurs, malgré leur importance économique sont de gros consommateurs d'eau. Suite à cela, les réserves en eau sont devenues de plus en plus limitées. La gestion et la répartition des ressources en eau entre le secteur agricole, le secteur industriel et l'usage domestique, constituent alors une problématique prioritaire pour le développement durable dans toute la région. Vu l'importance de ce sujet, plusieurs recherches (thèses, séminaires, rapports...) ont été menées sur la gestion des ressources en eau au Cap Bon (Gaaloul, 2008; Zghibi, 2007; Benalaya et al., 2009, Ghariani 2005, Gafsi et ben Haj, 2009...). L'objectif étant toujours, de trouver le meilleur moyen de gestion rationnelle de ces ressources qui s'épuisent de plus en plus mettant en danger le secteur agricole qui représente l'activité principale du Cap Bon et qui est considéré selon les dernières études comme étant le plus grand consommateur d'eau. Pour cela, les mêmes questions se posent « Le Cap Bon est-il capable de satisfaire les besoin d'eau de l'agriculture à long termes »? «L'eau comme élément vital multifonctionnel intervient t-il dans le processus et les mécanismes de la gestion différentielle des paysages du Cap Bon? ». Afin d'atteindre ces objectifs, plusieurs modèles de gestion ont été le résultat des recherches effectuées dans beaucoup de régions au Cap Bon: La plaine de Grombalia, la côte orientale du Cap Bon entre Kélibia et Béni khiar (Zghibi et al., 2009), les lagunes de Maamoura, Tazarka et Korba (Baccar et al., 2001), les bassins versants du Lebna et de Kamech (OMERE, 2010)... Bien que ces modèles aient pu rapporter de nouvelles méthodes de gestion intégrée des ressources en eau au Cap Bon, on remarque qu'ils sont insuffisants et que les mêmes problèmes se posent: insuffisance, mauvaise répartition, mauvaise exploitation, gaspillage, pollution... La gestion de la demande en eau constitue alors un axe principal de la politique générale de l'eau dans le futur,afin de maitriser la consommation au niveau des différents secteurs, surtout celui de l'agriculture, gros consommateur de ressources en eau (environ 80\%), et de retarder aussi longtemps que possible l'échéance du recours aux ressources en eau non conventionnelles (eaux usées traitées et eaux dessalées). Néanmoins, L'analyse de la question hydraulique ne peut pas se résumer à des données purement techniques et économiques. L'eau raconte la société. Les facteurs sociaux et politiques sont aussi déterminants (Mutin, 2009). Les outils de cette gestion doivent alors se baser essentiellement sur l'économie, la valorisation et la rationalisation de l'utilisation de l'eau non seulement comme une valeur économique mais aussi comme une valeur environnementale et paysagère de forte importance. Il est nécessaire alors de développer d'autres méthodes plus efficaces et surtout applicables, équitablement, dans toutes les villes du Cap Bon. La bonne gouvernance reste toujours la meilleure solution pour gérer durablement les ressources en eau. 


\section{Présentation du site}

\subsection{Localisation géographique de la zone d'étude}

La région du Cap Bon est une presqu'ile située à l'extrême Nord Est de la Tunisie. Orientée Sud-ouest à Nord-ouest, elle forme une avancée de $90 \mathrm{~km}$ vers le $\mathrm{NE}$ et s'étend sur $300 \mathrm{Km}$ de côtes ce qui représente le $1 / 5$ du littoral tunisien. Par contre, elle ne représente pas une zone de transition pour la Tunisie puisqu'elle n'est aucunement un lieu de passage entre le Nord et le Sud du pays, mais, son emplacement stratégique lui a permis d'être la terre de jonction entre les deux rives septentrionale et méridionale de la Méditerranée.

Le gouvernorat de Nabeul est la région administrative qui correspond géographiquement à la presqu'île du Cap Bon. C'est une étendue qui occupe une superficie d'environ $2840 \mathrm{~km}^{2}$ soit $1.8 \%$, de la superficie du pays, bordée d'une frange littorale de $200 \mathrm{~km}$ et possédant une population de plus de 787920 habitants (INS, 2014). En 2012, la région compte 16 délégations subdivisées en 24 municipalités et plusieurs conseils ruraux.C'est une région très urbanisée à cause de sa proximité de la capitale et de sa banlieue bien industrialisée et par sa localisation côtière à l'origine d'une activité touristique importante. Au cours de la période punique, cette région a connu un développement remarquable de l'activité agricole. Cette prospérité a attiré les Siciliens et les romains qui sont venus s'y implanter. Du côté urbain, la région se caractérise par l'essor de nombreuses villes littorales qui ont un rôle très important dans le dynamisme économique. Malgré la pression de l'urbanisme exercée sur les terres agricoles et les problèmes d'affectation des ressources en eau, elle demeure une des régions les plus fertiles et les plus riches sur le plan agricole. De point de vue ressources en eau, le Gouvernorat de Nabeul possède des ressources relativement importantes en eau et en sol et doit son développement agricole au savoir-faire d'une population maîtrisant relativement bien la technique d'irrigation, de l'arboriculture, du maraîchage et de l'élevage.

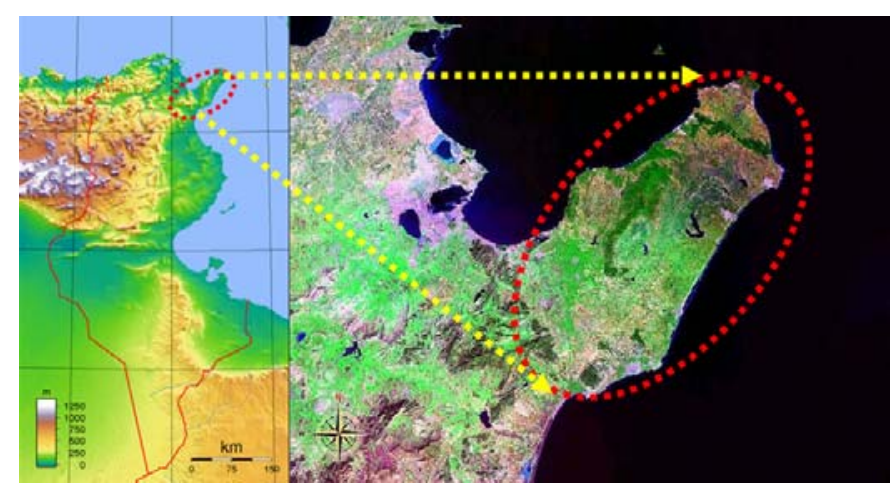

Figure 1: Localisation de la zone d'étude 
Le Cap Bon est riche en ressources hydrauliques (figure $n^{\circ} 2$ ). Elles proviennent surtout des eaux souterraines $\left(180 \mathrm{Mm}^{3} / \mathrm{an}\right)$, notamment sur le flanc oriental de Djebel Abderrahmane, sur la côte orientale et septentrionale $(60 \%$ des potentialités de la région) et dans les plaines de Grombalia ( $20 \%$ des potentialités) et d'El Haouaria (Gafsi et Ben Haj; 2009). Les bassins versants les plus importants sont du sud-ouest au nord est respectivement ceux d'oued Mornaguia, oued Laâbid, oued Zogag et oued El Magaiez.

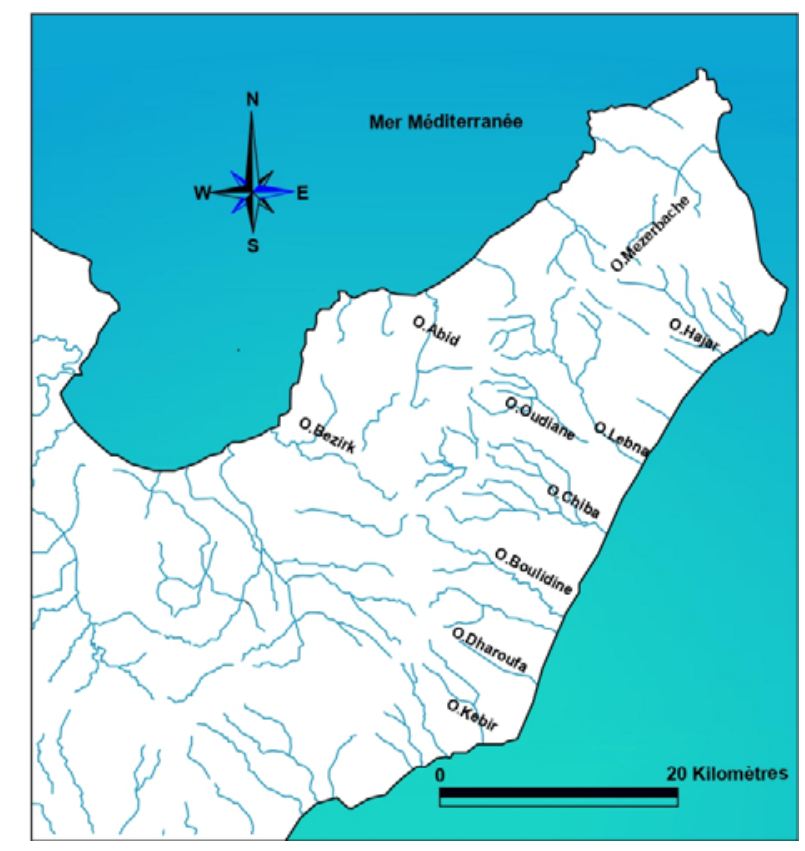

Figure 2: Réseau hydrographique dans la région du Cap Bon

Le cours d'eau le plus important est celui d'oued Bézikh et d'Laâbid (tableau $\mathrm{n}^{\circ} 1$ ). Ce dernier dont les affluents se caractérisent par leur intermittence devient permanent dans son cours inférieur. Un grand barrage vient d'être édifié sur son cours, la surface de l'aire de retenue est de 120 hectares, la capacité de la retenue est estimée à $106 \mathrm{~m}^{3}$. Cette retenue destinée à approvisionner des périmètres irrigués privera néanmoins son cours inférieur de l'écoulement permanent d'eaux douces mettant en danger les habitats naturels et la diversité biologique de l'oued et de l'estuaire (APAL, 2003). Bien qu'importantes, les eaux souterraines n'arrivent pas à faire face à l'accroissement rapide de la demande, tant dans le secteur agricole (plus de $80 \%$ de la demande) que dans le secteur touristique (800 1/jour/touriste) et industriel (Gafsi et Ben Haj; 2009). 
European Journal of Social Law

Tableau 1: Caractéristiques des oueds au Cap Bon (APAL, 2003)

\begin{tabular}{|l|l|l|l|}
\hline Oueds & Localisation & Bassin versant & Apport/an \\
\hline Oued Bézikh & Korbous & $157.0 \mathrm{~km}^{2}$ & $4.0 \mathrm{~mm}^{3}$ \\
\hline Oued Laabid & Centre du Cap Bon & $81.0 \mathrm{Km}^{2}$ & $6.0 \mathrm{~mm}^{4}$ \\
\hline Oued El Magaiez & Zaouiet el magaiez & $102.0 \mathrm{Km}^{2}$ & $2.0 \mathrm{~mm}^{5}$ \\
\hline Oued Hajjar & Kélibia & $80.2 \mathrm{Km}^{2}$ & $6.3 \mathrm{~mm}^{6}$ \\
\hline Oued Tafekhsit & Menzel yahya & $109.2 \mathrm{Km}^{2}$ & $9.9 \mathrm{~mm}^{7}$ \\
\hline Oued Lebna & Lebna/menzel horr & $205.0 \mathrm{Km}^{2}$ & $30.0 \mathrm{~mm}^{8}$ \\
\hline Oued Chiba & Korba/Mida & $204.0 \mathrm{Km}^{2}$ & $13.4 \mathrm{~mm}^{9}$ \\
\hline Oued Bouliedin ou Korba & Korba & $182.4 \mathrm{Km}^{2}$ & $8.0 \mathrm{~mm}^{10}$ \\
\hline
\end{tabular}

\subsection{Données climatiques et géologiques de la région du Cap Bon}

\subsubsection{Précipitation moyenne}

Les précipitations représentent le paramètre climatique le plus important, notamment dans les zones connues par une puérile en eau. Elles constituent également le facteur essentiel du régime hydrologique.La plaine de la côte orientale appartient à une zone modérément pluvieuse. Elle reçoit une moyenne annuelle qui varie de $400 \mathrm{~mm}$ au niveau de la station de Nabeul, à $442 \mathrm{~mm} / \mathrm{an}$ à Korba et 485 $\mathrm{mm} / \mathrm{an}$ à Kelibia (figure $\mathrm{n}^{\circ}$ ) ).

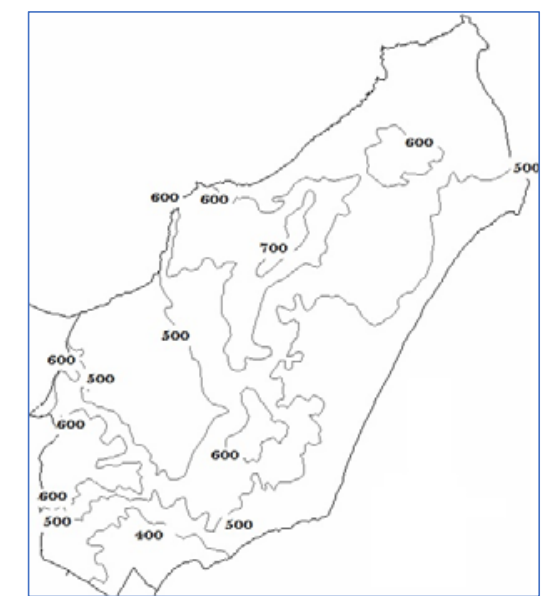

Figure 3: La carte pluviométrique annuelle du Cap Bon (Carte Agricole, 2016)

L'analyse des données pluviométriques relatives à une période de 44 ans d'observations de 1960 jusqu'à 2004, (figure $\mathrm{n}^{\circ} 4$ ), fait apparaitre une variabilité importante à l'échelle saisonnière et annuelle. En effet, le calcul du coefficient de 
variation montre que la pluviométrie sur la région de Korba est caractérisée par une irrégularité spatio-temporelle.

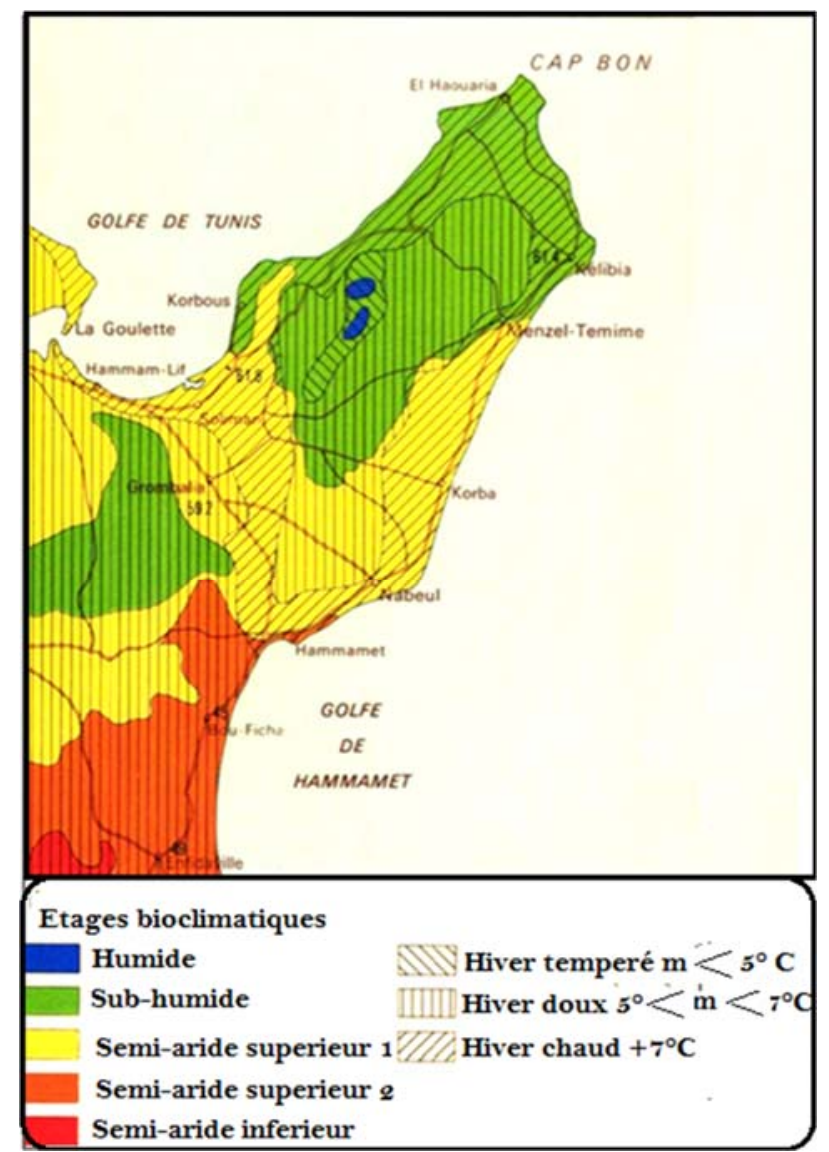

Figure 4: Etages bioclimatiques dans la région du Cap Bon (Gounot, 1965)

\subsubsection{Donnes géologiques}

La plaine de la côte orientale du Cap Bon correspond au flanc Est de l'anticlinal du Djebel Abderrahmane, dont les bordures sont occupées par les grès de l'Oligocène (formation Fortuna) et du Miocène (formation Oum Douil) et par les sables du Miocène supérieur (formation Somaa). Les produits d'érosion de cette structure constituent les dépôts aquifères du Pliocène constitue par les sables jaunes et blanc gris et du Quaternaire discordants sur le Miocène supérieur. Le synclinal de la Dakhla forme au cours du miocène renferme cette plaine côtière (figure $\mathrm{n}^{\circ}$ ). 


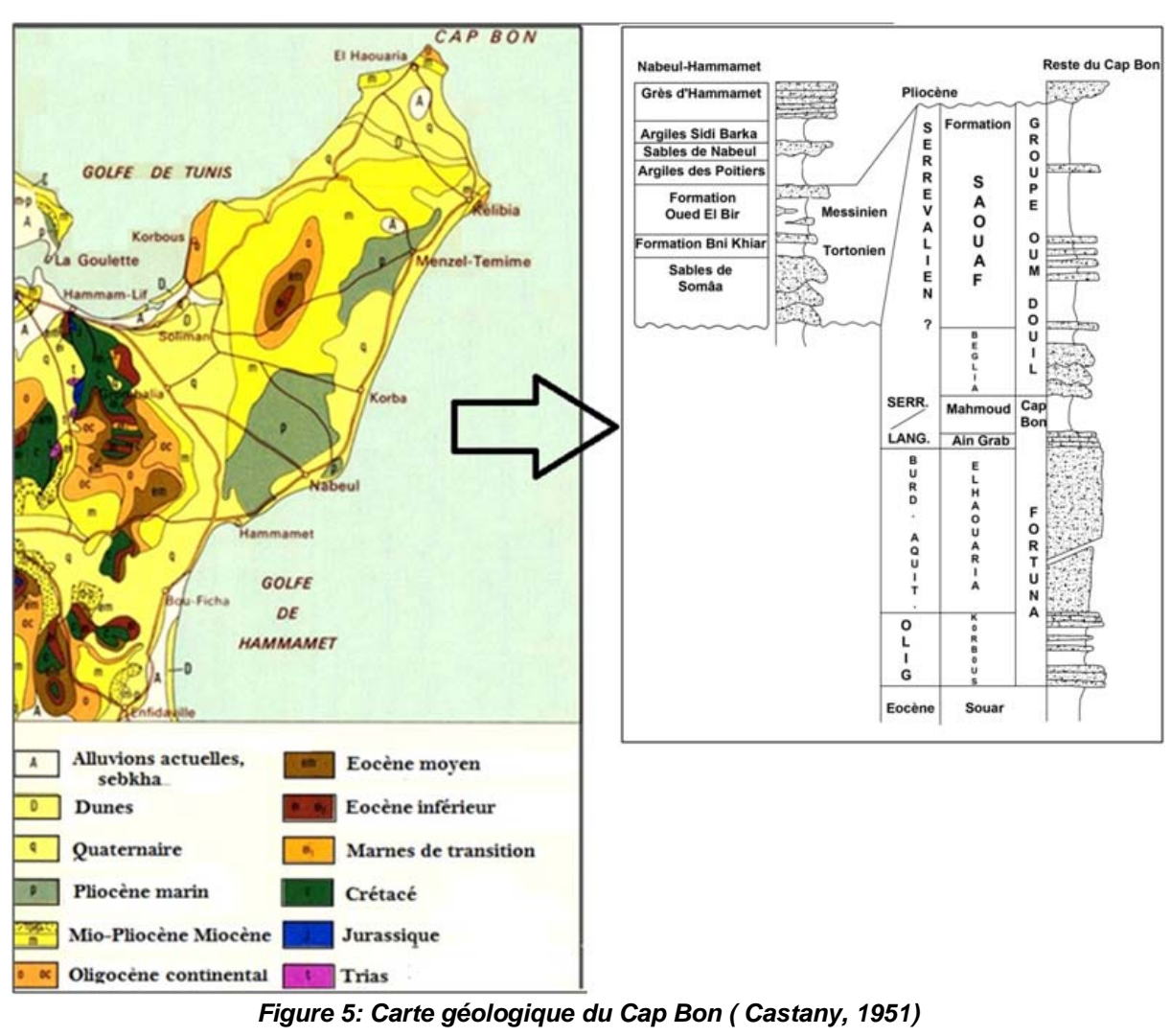

De ce fait, les grès des formations oligocènes et miocènes et les sables pliocènes et quaternaires constituent les seuls potentiels hydrogéologiques de la côte orientale. Pour la plaine d'El Haouaria, elle apparait comme une dépression enserrée par la mer des deux côtes Est et Ouest et par les affleurements de la formation d'Oum Douil (côté nord et sud). Sous le recouvrement dunaire et une couverture Quaternaire et un puissant dépôt Pliocène de $200 \mathrm{~m}$ environ constituent l'essentiel du remplissage de cette dépression d'El Haouaria qui constitue les réserves d'eau souterraine de la région (figure 5).

On peut rappeler du rôle important de la faille d'Azmour, située au Sud de la zone dunaire, dans l'alimentation hydrogéologique de cette plaine.

\section{Matériels et méthodes}

Vu l'insuffisance des recherches effectuées sur la gestion intégrée des ressources en eau et des zones humides au Cap Bon, nous avons vu nécessaire de faire un petit 
travail de terrain. Il s'agit d'une enquête effectuée auprès d'une dizaine d'habitants et d'agriculteurs des villes touchées par les conflits d'usage des ressources en eau et aussi par la défiguration de leurs paysages suite à l'urbanisation et à la pollution. L'objectif essentiel était de bien comprendre les causes de ces conflits, le degré de conscience de la population locale de la nécessité de préserver leurs ressources ainsi que le paysage d'eau qui reflète l'identité de chaque ville du Cap Bon.

Comme terrain d'étude, nous avons choisi la façade orientale du Cap Bon et plus précisément la région entre la ville de Kélibia et Béni Khiar qui possède l'une des plus importantes aquifères des plaines côtières Tunisiennes. Il s'agit d'une plaine qui s'étend sur une longueur de $45 \mathrm{Km}$ et qui se caractérise par des activités agricoles intenses menaçant ainsi la durabilité de la ressource en eau. Cette insuffisance de l'eau est justifiée aussi par l'absence de cours d'eau permanents malgré l'existence de nombreux oueds (Oueds Bouleddine, Lebna, Chiba, Abidis, El K'bhir et El H'jarr). Ainsi, la seule ressource en eau disponible sur place, provient des eaux souterraines peu profondes, ce qui justifie, selon les agriculteurs questionnés le recours au système du pompage pour l'irrigation et l'usage quotidien. Cette technique (le développement des pompages) a engendré de nombreuses conséquences à savoir la surexploitation de l'aquifère et l'avancé accélérée du biseau salé (Zghibi et al., 2009).

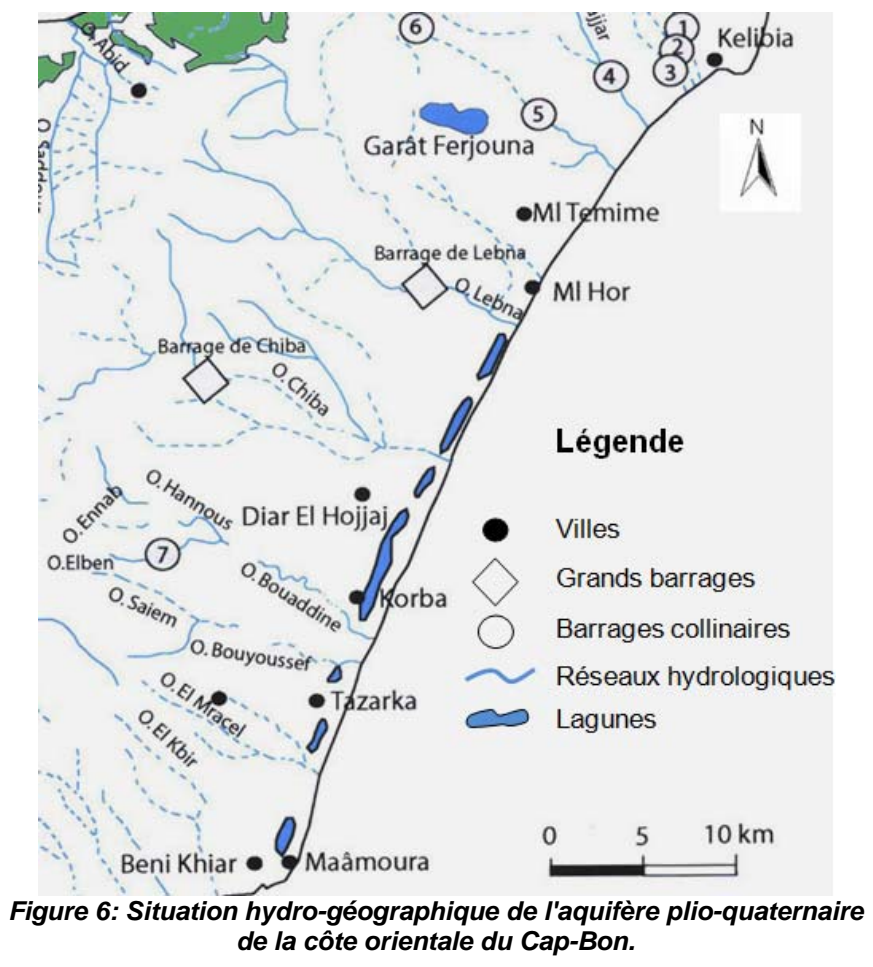


Selon le témoignage de la majorité des agriculteurs enquêtés, la zone d'étude souffre de l'absence d'une ressource en eau suffisante (entre le Sebkha et les agglomérations urbaines avoisinantes), de même, l'eau existante se trouve salée et vu la non productivité de sol et l'interdiction de bâtir (selon le plan d'aménagement municipal), la région n'a plus de valeur et se trouve marginalisée. Les agriculteurs déclarent aussi qu'il ya une insuffisance au niveau de système de sauvegarde qui a été conçu pour compléter les apports d'eau provenant des puits de surface. Cette insuffisance est justifiée par l'absence de programmes de réutilisation directe des eaux usées traitées dans la zone et aussi de taux élevé de salinité de l'eau existante. D'un autre côté, d'autres agriculteurs réclament le coût élevé de l'eau et le manque d'information et de sensibilisation sur la bonne utilisation de l'eau disponible. Afin de résoudre le conflit d'usage de l'eau, des solutions ont été proposées par le gouvernement telles que l'alimentation de la nappe par les eaux du Nord, mais seulement en été, chose qui n'intéresse pas les agriculteurs qui ont besoin de l'eau en été surtout pour la culture de tomate. Les interventions de l'état (périmètre de sauvegarde, recharge de la nappe, incitations financières...) n'ont pas réussi à résoudre le problème des agriculteurs qui se plaignent de la pollution (rejet des eaux usées dans les lagunes), de la situation des terres limitrophes des lagunes et des personnes qui les exploitent sans avoir un certificat de propriété. Cette absence d'informations, de dialogue et de communication entre les agriculteurs et les pouvoirs publics a provoqué la naissance de nombreux conflits qui ont empêché la gestion durable des ressources en eau.

\section{Résultats}

\subsection{La gestion des ressources disponibles}

Selon les dernières études effectuées sur l'eau et la majorité des agriculteurs enquêtés, le Cap Bon est considéré comme une région pauvre en ressources hydrauliques. Les bilans hydriques établis prouvent qu'aujourd'hui, la région du Cap Bon est devenue de plus en plus dépendante aux transferts importants d'eau en provenance du Nord du pays (Chaibi et al, 2004). Selon la figure ci-dessous, la demande estimée pour l'année 2020 dépasse largement les offres surtout durant les mois d'été ou les taux d'irrigation sont à leur maximum ce qui nécessite la planification d'autres apports extérieurs importants pour éviter les risques de l'insuffisance des ressources en eau (figure 2). 


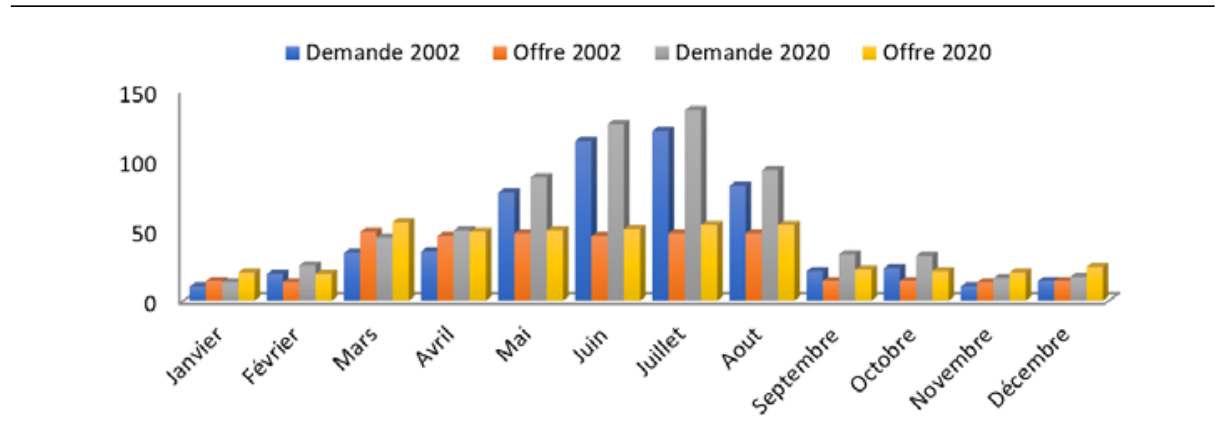

Figure 7: Evolution de l'offre et de la demande en eau de l'année 2002 à 2020 au Cap Bon, Gafsi et Ben Haj, 2009

Suite à cette insuffisance des ressources en eau, l'agriculture du Cap Bon commence à connaître de profondes mutations: déclin relatif de l'agrumiculture face au problème de surexploitation de la ressource en eau, développement du maraîchage intensif grâce à la vulgarisation des motopompes et à l'importance grandissante des primeurs, regain d'intérêt pour la production viticole. En effet, le secteur des agrumes qui a toujours figuré au premier rang dans l'agriculture du Cap Bon (contribution de $85 \%$ à la production nationale, source de revenus pour 8.730 agriculteurs) se trouve menacé par l'irrégularité et le tarissement des ressources hydriques et la salinité des nappes phréatiques. Un problème d'autant plus prégnant qu'il fallait recourir aux eaux du Nord pour sauver la forêt agrumicole dans le Cap Bon. Selon les agriculteurs « Les quantités d'eau administrées varient de 6000 à $10000 \mathrm{~m} \mathrm{3/ha/an} \mathrm{»,} \mathrm{explique} \mathrm{un} \mathrm{fellah}$ qui ajoute que « des groupements de développement agricole ont été créés pour répartir l'eau entre les agriculteurs surtout durant la période d'irrigation qui s'étale de mois de mars à octobre, durant cette période, la demande en eau agricole ne cesse d'accroître pour irriguer une agriculture tournée vers l'exportation ». Certains fellahs estiment que le coût d'un m3 d'eau d'irrigation devient de plus en plus cher. Pour d'autres « Un hectare revient à 400 dinars soit $10 \%$ du coût réel de la production. Mais en fait l'eau participe réellement à $60 \%$ de la production. N'ayant pas une véritable conscience de la valeur économique de l'eau, les usagers ont souvent tendance à gaspiller l'eau et à avoir recours à des techniques et des pratiques agricoles peu efficientes. «Il faudrait peut-être augmenter les tarifs d'eau en vue d'encourager progressivement les économies d'eau » affirme l'un des fellahs questionnés qui se plaint aussi de l'état médiocre et du manque d'entretien des canalisations conduites d'eau qui ont contribué aussi au gaspillage de l'eau d'irrigation.

\subsection{Impact environnemental et la destruction des écosystèmes des zones humides}

Suite au développement économique qu'a connu le Cap Bon ces dernières années, les aménagements touristiques, urbains, agricoles et industriels effectués ont impacté négativement les paysages naturels de toutes les villes littorales du Cap Bon 
et en particulier les villes faiblement touristiques telles que la ville de Korba, de Tazarka, et d'El Maamoura (Romdhane et Missaoui; 2001). Ces aménagements ont pollué les lagunes et leurs alentours constitués essentiellement de terres agricoles. A cet égard, les habitants de ces villes et les agriculteurs qui possèdent des terrains limitrophes à ces zones humides, se plaignent énormément de l'étalement urbain incontrôlé, de la pollution sous ces différentes formes (le déversement des eaux usées ou autres; les rejets des usines de conserves dont le volume est considérable; les rejets d'autres industries telles que le délavage des jeans et autres produits textiles; l'accumulation volontaire ou involontaire des déchets divers) qui impacte négativement la qualité des eaux des puits existants considérés comme étant le seul moyen d'irrigation disponible. D'un autre côté, les constructions anarchiques ont défiguré le paysage de ces milieux humides riches en faune et flore et ont minimisé de son attraction touristique.

« Malgré que j'habite près de la mer, le paysage de la lagune avec le cumul de déchets et les constructions dépourvues de tout côté esthétique, me dérange énormément. Il faut que la commune de la ville de Korba trouve une solution pour interdire de bâtir tout près des zones humides et aussi d'interdire aux habitants et aux usines de jeter leurs déchets » affirme l'un des habitants de la ville de Korba et rajoute que « C'est une très grande nuisance à l'identité et la réputation de la ville qui commence de plus en plus à perdre ses beaux paysages littoraux et par la suite sa capacité d'attirer les touristes, de ce fait, l'assainissement et l'aménagement de la lagune de Korba sont des opérations considérées comme urgentes ».

\subsection{La valeur socio-économique de l'eau et les différents usages}

L'eau se place toujours au cœur de l'activité économique et sa gestion s'effectue selon les principaux usages, agricole, industriel, touristique et domestique. Cette multiplication des usages conjuguée à l'augmentation des besoins dans le cadre d'une gestion cloisonnée ne manque pas d'engendrer les premiers conflits (Ghiotti, 2006).Avec plus que $2060 \mathrm{~km}^{2}$ de terres cultivées (A.U.D.E.C, 1996) le secteur agricole au Cap Bon représente une part prédominante dans l'économie de la région et consomme beaucoup d'eau (Sethom, 1977). C'est le plus grand consommateur d'eau puisqu'il utilise $80 \%$ des ressources en eau et ce pourcentage devrait encore augmenter afin d'assurer les demandes en alimentation de l'accroissement démographique (Gafsi et Ben Haj; 2009).D'un autre côté, le secteur touristique constitue un axe majeur de la politique de développement et d'aménagement du territoire. La demande en eau par ce secteur stresse énormément les ressources disponibles et le système d'approvisionnement surtout en été lorsque les besoins en eau pour l'irrigation sont très importants. Selon les dernières statistiques (voir tableau 2), la consommation de l'eau à usage touristique a augmenté de près de $30 \%$ contre $6 \%$ pour l'eau à usage domestique qui consomme aussi une grande quantité d'eau mais elle reste très faible si on la compare aux autres usages (Gafsi et Ben Haj; 2009). 
La ville de Hammamet est classée parmi les villes les plus consommatrice en eau avec environ 4.370 mille $\mathrm{m}^{3}$ pour son secteur touristique fort développé et 3.275 mille $\mathrm{m}^{3}$ pour l'usage domestique. Vient par la suite, la ville de Nabeul qui constitue le deuxième pôle touristique, et puis la ville de Kélibia qui est considérée comme une ville moyennement touristique (tableau $\mathrm{n}^{\circ} 2$ ).

Tableau 2: Consommation de l'eau potable selon l'usage (SONEDE, 2008)

\begin{tabular}{|l|l|l|l|l|l|l|}
\hline \multirow{2}{*}{ Délégation } & \multicolumn{3}{c|}{ Usage touristique } & \multicolumn{3}{c|}{ Usage domestique } \\
\cline { 2 - 8 } & $\mathbf{2 0 0 6}$ & $\mathbf{2 0 0 7}$ & $\mathbf{2 0 0 8}$ & \multicolumn{1}{c|}{$\mathbf{2 0 0 6}$} & $\mathbf{2 0 0 7}$ & $\mathbf{2 0 0 8}$ \\
\hline Nabeul & 170 & 190 & 400 & 2.333 & 2.689 & 2.675 \\
\hline Dar Chaabane Fehri & 49 & 43 & 232 & 1.018 & 1.050 & 1.030 \\
\hline Beni Khiar & - & - & - & 977 & 1.175 & 1.170 \\
\hline Korba & 21 & 22 & - & 1.540 & 1.564 & 1.540 \\
\hline Bouargoub & - & - & - & 393 & 400 & 370 \\
\hline Hammamet & 3.895 & 3611 & 4.370 & 2.930 & 3.284 & 3.275 \\
\hline El Mida & - & - & - & 379 & 398 & 420 \\
\hline Menzel Temime & 145 & 150 & 120 & 1.245 & 1.365 & 1.540 \\
\hline Kelibia & 265 & 280 & 300 & 1.440 & 1.395 & 1.640 \\
\hline Hammam El Guezaz & 10 & 11 & 12 & 275 & 275 & 330 \\
\hline El Haouaria & 170 & 180 & 190 & 620 & 670 & 780 \\
\hline Takelsa & - & - & - & 142 & 165 & 125 \\
\hline Soliman & 37 & 50 & 52 & 1.053 & 1.100 & 1.166 \\
\hline Menzel Bouzelfa & - & - & - & 628 & 770 & 799 \\
\hline Beni Khalled & - & - & - & 839 & 810 & 875 \\
\hline Grombalia & - & - & - & 1.645 & 1.470 & 1.555 \\
\hline Total & 4.762 & 4.537 & 5.676 & 17.457 & 18.580 & 19.290 \\
\hline
\end{tabular}

\subsection{Conflits d'usages d'une ressource limitée}

Malgré les importantes réalisations de l'Etat qui a construit une dizaine de barrages sur les oueds (barrages Lebna, Chiba, Bézikh...) et mis en place des systèmes d'infiltration d'eaux usées traitées dans l'aquifère, les eaux de surface (160 Mm3 mobilisables) et les eaux profondes (29 Mm3 exploitables annuellement), restent toujours insuffisantes. Au cours del'année (2007-2008), la consommation de l'eau à usage touristique a augmenté de près de $30 \%$ contre $6 \%$ pour l'eau à usage domestique. Les ressources en eau sont surexploitées (taux d'exploitation proches des $200 \%$ dans la plaine de Grombalia et d'El Haouaria) et les économies d'eau limitées étant donné que les systèmes d'irrigation sont déjà optimisés (goutte à goutte). Les bilans hydriques montrent que le Cap Bon ne peut vivre aujourd'hui sans les transferts importants d'eau (77 Mm3/an) en provenance du Nord du pays; ceux-ci devraient augmenter dans l'avenir. Cette situation pose au niveau national le problème de l'affectation optimale de ressources limitées. La question qui se pose est de savoir quelles sont les activités qui justifient, eu égard à leur rendement élevé, un transfert au détriment d'autres 
régions ou qui pourraient supporter le coût d'un traitement par des procédés de dessalement. Il est également important de souligner que des efforts significatifs d'économie d'eau seront à consentir dans tous les secteurs. C'est au niveau de la demande agricole que les efforts les plus importants sont à fournir mais également au niveau de la demande touristique et domestique $(3 \%=$ taux de croissance annuel de la demande), industrielle ( $1,5 \%=$ taux de croissance annuel de la demande) (Gafsi et Ben Haj; 2009). Mais il faut s'attendre à une demande d'eau beaucoup plus importante que le simple accroissement démographique en raison des mutations économiques et sociales qu'enregistre la société: l'augmentation des superficies irriguées et la croissance urbaine (Mutin, 2009).

\subsubsection{Effet du secteur touristique et de l'urbanisation sur la gestion de l'eau}

$\mathrm{Au}$ Cap Bon, les 135onsummation135 du secteur touristique et de l'étalement urbain se font également ressentir, autant en termes de 135onsummation d'espace, parfois 135 onsummati, que de ressources naturelles, et principalement l'eau qui est surexploitée $\mathrm{du}$ fait d'une surconsommation estivale dans les 135onsum d'hébergement touristique et par la population urbaine qui connaît un boom lors des grandes vacances. La production d'eaux usées domestiques connaît également un accroissement 135 onsummati 135 onsum la haute saison, mettant à mal l'efficacité des stations de traitement. Sur le plan environnemental, le développement touristique et urbain a réduit la perméabilité des sols. Il a 135onsumma ainsi une augmentation des volumes d'eau à évacuer et une diminution des temps de concentration. En outre, il s'agit d'un tourisme de masse qui accroît la pression sur les ressources en eaux et génère des quantités importantes de déchets solides et de rejets d'eaux usées qui seront évacuées en mer après 135onsummat (Ghariani, 2005). Notons par exemple le cas de la ville de Hammamet qui abrite le grand 135ons touristique au Cap Bon, la station balnéaire de Yasmine Hammamet. Pour la population additionnelle, estimée à 72.000 habitants à l'horizon 2020, les besoins en eau ont été calculés sur la base de 150 1/personne/jour pour le milieu urbain et 100 1/personne/jour pour le milieu rural. La 135 onsummation d'eau de la zone est estimée à $12 \mathrm{~mm} 3$ don't 8 pour la zone touristique (sur la base de 600 1/jour/lit, ratio inférieur au ratio méditerranéen estimé à 800 l/jour/lit). Elle se situe aujourd'hui aux environs de $6 \mathrm{~mm} 3$ pour l'ensemble de la conurbation. Des solutions devront donc être trouvées pour l'approvisionnement de la zone. Sachant que les ressources en eau potable proviennent à $90 \%$ du canal des eaux $\mathrm{du}$ Nord dans cette zone, faudra-t-il prévoir de nouveaux transferts, procéder au dessalement de l'eau de mer, diminuer la superficie des terres agricoles irriguées? (Gafsi et Ben Haj, 2009).

\subsubsection{Effet de l'agriculture et des pratiques agricoles sur la qualité de l'eau}

Au Cap Bon, l'agriculture qui est l'activité initiale de la région, consomme énormément de l'eau surtout au niveau des plaines où les conditions physiques et socio-économiques favorisent l'intensification des cultures. Cette intensification est 
favorisée essentiellement par l'évolution des pratiques agricoles à travers l'utilisation excessive d'engrais chimiques et organiques et des traitements phytosanitaires. Dans les villes agricoles du Cap Bon, notamment la ville de Korba, de Menzel Temim et d'El Haouaria, les agriculteurs n'hésitent pas à employer des substances phytosanitaires notamment, pour protéger leurs cultures des plantes adventices, des dégâts des prédateurs et des attaques des organismes pathogènes.

Selon un agriculteur de la ville de la ville de Korba «il est nécessaire d'utiliser les fertilisants afin de rendre l'agriculture plus rentable surtout qu'il y a une insuffisance d'eau et les conditions climatiques sont devenues de plus en plus difficiles $»$. A cet égard, l'utilisation des engrais chimiques a augmenté par hectare labourable (elle est passée de $5 \mathrm{~kg}$ au début des années soixante pour atteindre $22 \mathrm{~kg}$ en 2001) (Ghariani, 2005). L'inexistence des mesures agro-environnementales efficaces constitue la raison principale de cette augmentation incontrôlée de l'utilisation des produits chimiques.

Selon l'enquête que nous avons menée, c'est la tradition culturale des fellahs qui a été déterminante dans le développement de la région. « Le niveau d'éducation des agriculteurs est généralement faible et leurs pratiques agricoles fondées sur les connaissances locales traditionnelles ne sont pas toujours efficaces et tendent à dégrader le sol, et au final, à faire perdre de sa durabilité et de sa productivité » a affirmé l'un des habitants de la ville de Korba.

Dans cette ville, plusieurs stratégies ont été observées. Certains agriculteurs ont recours au système d'irrigation de goutte à goutte ou au pompage privé, en particulier pour le maraîchage ou les cultures fourragères destinées à l'élevage bovin laitier; d'autres préfèrent retourner aux cultures pluviales, notamment les céréales, et à l'élevage ovin.Bien que le matériel d'irrigation de goutte à goutte a limité le gaspillage des eaux, mais on trouve qu'il a favorisé l'apport des engrais et la salinisation des sols (diminution de la frange de lessivage). De même, la situation foncière caractérisée par le morcellement des parcelles a accentué la pression sur les ressources en eau (utilisation excessive en été) et en sol.

\section{Discussion}

\subsection{Intervention de l'État en matière de gestion des ressources en eau.}

Vu la vulnérabilité relativement élevée des ressources en eau en Tunisie et en particulier au niveau de la région du Cap bon, plusieurs politiques et stratégies de gestion de l'eau ont été mises en place. Selon le rapport national sur le secteur de l'eau, les principales politiques nationales sont les suivantes (Mekki, 2006): le développement et la mobilisation des ressources en eau disponibles afin d'atteindre un taux de mobilisation de $95 \%$; la gestion intégrée des ressources en eau, en particulier l'amélioration du transfert des volumes excédentaires des périodes pluvieuses pour les périodes de sécheresse; les économies d'eau et la maîtrise de la 
demande dans tous les secteurs; la poursuite du développement des ressources en eau non conventionnelles, par le biais de l'expansion de l'usage des eaux usées traitées dans l'agriculture et des eaux saumâtres issues du dessalement dans tous les secteurs; la protection des ressources en eau contre la pollution et la surexploitation.

Comme objectif principal, l'alimentation en eau potable est prioritaire afin d'assurer l'équité entre les zones rurales pas encore desservies et les zones approvisionnées par la SONEDE. Par contre le tourisme et industrie sont réaffirmés comme deux activités majeures dont les prélèvements ne doivent pas être limités. Il s'agit alors d'une politique qui vise la gestion de la demande, tout en cherchant à développer encore mieux la ressource (Treyer, 2000).

Afin de répondre à ces politiques, trois grandes stratégies ont été mises en place. La première stratégie est la stratégie décennale de mobilisation des ressources en eau (1990-2000). Cette stratégie a été élaborée et mise en œuvre afin de fournir un contrôle intégré des sources d'eau potentielles. Elle a comme priorité majeure l'augmentation de l'offre. La deuxième stratégie est la stratégie complémentaire (2001-2011). Cette stratégie tente de réaliser les objectifs à long terme afin d'assurer l'équilibre durable de la demande et des ressources en eau disponibles. Elle se compose en partie de mesures similaires à celles de la stratégie de mobilisation, en vue de parvenir à un taux de mobilisation de $95 \%$. En outre, la stratégie met l'accent sur les mesures de régulation entre les années sèches et humides, les mesures de conservation des sols et des eaux et la recharge des aquifères. La troisième stratégie est la stratégie à long terme (jusqu'à 2030). Celle-ci repose principalement sur les plans directeurs des eaux. La stratégie se compose d'un nombre élevé d'études et de programmes de recherche qui ont pour objectif d'assurer la durabilité des moyens de gestion des ressources en eau à long terme.

\subsection{Cadre institutionnel}

Actuellement, le système politique de gestion de l'eau reflète les changements des dix dernières années. L'administration et le Ministère de l'Agriculture gardent leur importance, mais un Ministère de l'Environnement et de l'Aménagement du Territoire a vu le jour, ainsi qu'un Comité National de l'Eau encore balbutiant censé rassembler administration et usagers. Des sociétés publiques autonomes sont chargées de fonction telles que l'alimentation en eau potable (SONEDE), l'exploitation du canal et des adductions du Nord (SECADENORD), ces deux sociétés sous la tutelle du Ministère de l'Agriculture, et un Office chargé de la gestion et de l'exploitation des ouvrages d'assainissement (ONAS). Les Offices de Mise en Valeur Agricole ont été remplacés en 1989 par les Commissariats au Développement Agricole, qui représentent un début de décentralisation. La politique actuelle s'est aussi orientée vers plus de d'implication des usagers avec la création des Associations d'Intérêt Collectif pour l'alimentation en eau potable rurale, les périmètres publics irrigués, et peut-être bientôt pour les usagers des nappes phréatiques subissant la surexploitation. Ces AIC sont soumises à la tutelle d'un 
Groupement d'Intérêt Hydraulique, équivalent régional du Comité National de l'Eau (Treyler, 2000; Bruno Romagny et Christophe Cudennec, 2006; Al Atiri, 2006).

\subsection{Les ouvrages de conservation d'eau: forte valeur paysagère et patrimoniale}

Selon Honeggaer (2008), Chaque espace hydraulique est un espace social traversé par de multiples logiques d'ordre géographique, économique mais aussi politique, symbolique et idéologique.

Les aménagements de conservation des eaux et des sols sont importants à considérer à la fois pour caractériser le milieu et pour appréhender le paysage et participer à sa construction. Ils orientent la dynamique actuelle du milieu et en particulier celle de l'eau. En outre, ils structurent le paysage, le diversifient, le cloisonnent, accentuant ou non le parcellaire.

Ces aménagements jouent le rôle d'une explicative: ils permettent de retrouver l'articulation logique d'un paysage caractérisée par une juxtaposition ou une succession de dynamiques différenciées (Boyer, 2006).

Dans la région du Cap Bon, les ouvrages de conservations des eaux et des sols sont très anciens et remontent presque à la naissance de l'agriculture dans l'antiquité. Ces ouvrages construits afin de préserver l'eau et assurer sa distribution ont fortement marqué le paysage du Cap Bon et leur développement semble avoir touché et structuré son littoral.

L'apport des civilisations antiques particulièrement romaines a fortement marqué le domaine hydraulique au Cap Bon (infrastructures, pratiques de gestion de l'eau...). Ces derniers perfectionnèrent de nombreuses techniques traditionnelles. De nombreux vestiges d'ouvrages hydrauliques persistent jusqu'à maintenant et constituent un héritage culturel de forte importance. A leur tour, Les carthaginois avaient élaboré un système de canaux d'irrigation qui donnait au paysage l'aspect de jardin parcourus d'eau courante. Il ya eu la construction d'un important réseau d'aqueducs pour l'alimentation en eau (Temple-Boyer, 2006). Des ruines d'ouvrages hydrauliques, situées à proximité des points d'eau, attestent des travaux d'aménagement dont la plaine a fait l'objet depuis les temps les plus anciens. Parmi les vestiges les plus remarquables, on trouve les aqueducs romains à l'Ouest de la ville de Grombalia et au Sud de Turki, deux importants bourgs fondés par les Andalous expulsés d'Espagne au début du XVIIème siècle (Abdelkafi, 2004). On peut distinguer trois types d'aménagement qui marquent le paysage en tant qu'éléments spatiaux fonctionnels: l'aménagement ponctuel (ouvrages de cours d'eau: les jessours, gabions...), l'aménagement linéaire (banquette, cordons de pierre, haies de cactus...) et l'aménagement aréolaires (reboisement forestiers, les parcelles du cactus). Cette typologie est intéressante de point de vue hydrologique (Temple-Boyer, 2006). 


\section{Recommandations et sensibilisation}

A travers les résultats et les données qu'on a pu collecter tout au long de ce travail, nous avons bien remarqué que malgré les efforts de l'état en matière de la gestion des ressources en eau et de la préservation de la qualité paysagère des zones humides, les agriculteurs des villes littorales du Cap Bon se plaignent toujours de l'insuffisance de l'eau suite au mauvais usage de la part du secteur touristique, du secteur industriel ainsi que des pratiques agricoles de certains d'entre eux qui au lieu de mieux gérer cette ressource limitée, contribuent eux aussi à sa pollution. Les habitants de ces villes se plaignent aussi de la défiguration de leur paysage suite aux rejets de divers déchets dans les lagunes et aux abords des oueds et suite aux constructions anarchiques éparpillées partout. Ils demandent une intervention urgente de la part de l'état afin de mettre fin à toutes atteintes à la beauté de leurs villes et de mieux gérer leurs ressources. Pour atteindre cet objectif et en se basant sur les principaux avis des personnes enquêtées, nous présentons ici quelques recommandations qui servent comme modèle de gestion différentielle du paysage et des ressources en eau dans les villes littorales du Cap Bon:

\subsection{La rationalisation de l'usage d'eau et l'exploitation de nouvelles ressources}

Tout d'abord un travail de sensibilisation et de rationalisation des différents utilisateurs des ressources en eau doit être effectué. L'état doit prendre en charge l'organisation des réunions et de conférences pour sensibiliser les agriculteurs à la meilleure manière d'utiliser et d'économiser l'eau. Pour cela la diversification de la culture et le changement de pratiques culturales sont conseillés. Comme propositions de bonnes pratiques, les agriculteurs peuvent pratiquer l'assolement afin de résoudre l'insuffisance de l'eau au niveau du périmètre de sauvegarde et peuvent penser aux cultures maraichères qui sont moins consommatrices en eau. De même, pour trouver des solutions à l'insuffisance de l'eau, l'alimentation de la lagune par les eaux usées traitées permet de maintenir un plan d'eau permanent et diminue aussi le taux de salinité. Par contre pour la recharge des puits de l'eau, il faut penser à mettre en place un réseau hydraulique. Il faut aussi penser à l'exploitation de nouvelles ressources pour faire face aux besoins croissants en eau et à l'urbanisation du monde.

Dans un contexte de besoins croissants, la mobilisation de nouvelles ressources, que ce soit des ressources classiques comme les eaux perdues dans les réseaux de distribution ou des ressources alternatives comme les eaux usées recyclées et les eaux de dessalement est un des moyens incontournables pour préserver les ressources d'eau disponibles. A cet égard, l'état doit sensibiliser les utilisateurs à la nécessité d'utiliser ces ressources non conventionnelles afin d'éviter la pénurie d'eau. Pour cela, il faut forger des modèles économiques capables de financer et 
d'entretenir correctement les infrastructures, de respecter des normes plus strictes, de fournir l'eau potable et l'assainissement à tous d'une manière équitable.

\subsection{La Redéfinition de la valeur de l'eau comme élément structurant du paysage}

L'eau joue un rôle majeur en matière de structuration paysagère pour cela elle doit être intégrée dans les processus de structuration de l'espace et de la société. Cette intégration se fait à travers la préservation des cours d'eau, et des zones humides contre la pollution et l'urbanisation anarchique qui a contribué à l'apparition des zones de friche urbaine à la périphérie des villes, où s'installent des communautés humaines privées de l'accès aux infrastructures et aux services publics essentiels. Ces zones humides doivent être alors mises en valeur, aménagées et intégrées dans la trame urbaine. La valeur patrimoniale accordée à ces paysages d'eau leur donne une nouvelle fonction et permet leurs préservations comme richesse naturelle de forte importance.

\subsection{L'intégration de la dimension paysagère dans les actions de lutte contre les ruissellements et les risques}

Il faut participer à la préservation de la qualité de l'eau, des écosystèmes aquatiques, des sites et des zones humides, ainsi qu'au développement des ressources en eau. Il faut concilier les besoins en eau dans les différents domaines d'activités tout en favorisant le renouvellement du cycle de l'eau. Dans les régions où les précipitations sont fréquentes, il faut prendre en compte une gestion raisonnée de l'assainissement en milieu urbain en intégrant des systèmes d'assainissement et de filtration naturelle, et éviter la saturation du réseau en créant des zones de rétention supplémentaire. Dans les réseaux agricoles, mieux tenir compte du relief et des espaces naturels pour ne pas désacraliser le paysage et préserver les zones humides... Les zones humides sont des espaces de transition entre la terre et l'eau et font parties des milieux les plus riches du monde en raison de leur richesse biologique et de leur fonctions naturelles.

En d'autre terme, l'intégration de l'approche paysagère et environnementale dans la gestion de l'eau peut et doit s'accompagner d'une urbanisation et d'une architecture des plus contemporaines afin d'aboutir à un projet au plus près des aspirations des futurs urbains.

Dans le domaine de l'eau, s'il s'agit toujours de participer à l'organisation d'un territoire qui souffre de la relation conflictuelle entre ses multiples fonctions (productives, touristiques, agricoles, urbanistiques, environnementales) pour l'utilisation et la gestion de ses ressources (fonciers, ressources en eau, biodiversité, paysages, etc.). Dans ce contexte, l'eau n'est plus uniquement un support mais un facteur de développement (Ghiotti, 2006). 
Afin d'améliorer ses stratégies de gestion et de préservations des ressources en eau au Cap Bon, l'état a besoin d'une démarche plus participative qui puisse associer l'ensemble des composantes de la société civile à la gestion des ressources, dans le cadre de la redéfinition des relations entre l'État et le secteur privé. Ceci nécessite l'élaboration de nouveaux dispositifs de gestion de l'eau afin de résoudre les conflits d'usages entre le secteur public et le secteur privé, tout en faisant face à la complexité croissante de l'approvisionnement en eau potable. Il en résulte une nouvelle génération de mesures de politique environnementale qui, prenant la forme de dispositifs hybrides, s'inscrit dans une démarche participative où interviennent, à côté des autorités publiques, des acteurs issus de la sphère marchande comme de la société civile (Makkaoui et Dubois, 2010).

\section{Conclusion}

Partout dans le monde et plus précisément dans les pays méditerranéens comme la Tunisie, la préservation des ressources et des paysages d'eau sont devenues ces dernières années un sujet de préoccupation majeure. Toutefois, leurs efforts pour la gestion de l'offre ont pu atteindre les limites en termes de qualité (défiguration des paysages suite à la pollution des lagunes, sebkhas et oueds et à l'urbanisation accrue) et de quantité (insuffisance suite à la mauvaise utilisation et répartition entre les différents secteurs économiques), comme nous le voyons de notre étude de cas du Cap Bon en Tunisie. Bien que cette région a développé de nombreuses stratégies de gestion de l'eau mais on assiste encore à de nombreux échecs au niveau de la gouvernance.

Par ailleurs, il y a une inégalité de partage des ressources en eau, entre le secteur agricole et les autres secteurs socio-économiques ce qui nécessité la mobilisation davantage de ressources, notamment en raison de la forte concurrence entre les secteurs, la convergence des intérêts, l'absence de partenariat effectif ainsi que l'absence d'un « lieu » ou de mécanismes de concertation effective, qui représentant autant de facteurs liés à l'absence de communication et de coordination efficaces. Par conséquent, les autorités publiques doivent renouveler leur structure de gouvernance à l'échelle régionale et nationale. Elles doivent développer une collaboration solide entre les différentes parties concernées et mettre en place des stratégies novatrices en matière de gestion des ressources en eau et des zones humides, dans un effort commun pour assurer une croissance économique durable et créer de la diversité. Un travail de sensibilisation des différents utilisateurs et surtout les agriculteurs de la nécessité de préserver les ressources en eau à travers la mise en place d'un système d'information et d'une plateforme de dialogue, s'avère nécessaire.

En conclusion, les ressources en eau ont une grande importance en termes de contribution au développement économique, à la protection de l'environnement et à l'aménagement du paysage. La mise en valeur paysagère des ressources et du paysage d'eau au Cap Bon et son intégration dans les politiques de gestion d'eau 
constitue une bonne stratégie de gestion qui peut contribuer à la résolution des conflits d'usage au Cap Bon et par la suite au développement durable de toute la région si un bon système de gouvernance sera mis en place.

\section{Référence bibliographique:}

- Abdelkafi, J., 2004. "Le vignoble du Cap Bon (Tunisie)" Les paysages culturels viticoles, p.139-141

- AL ATIRI, R., 2006. Evolution institutionnelle et réglementaire de la gestion de l'eau en Tunisie Vers une participation accrue des usagers de l'eau. Actes du séminaire Wademed, Cahors, France, 6-7 novembre 2006. Cirad, Montpellier, France

- APAL., 2003. Plan de gestion des sites d'Oued Laâbid et de Dar Chichou, Analyse des potentialités et des contraintes et proposition de scénarios de gestion, http://www.apal.nat.tn/.../digitalAssets/141_PG_La_bid-Chichou. p.26

- A.U.D.E.C. (1996) Atlas du Gouvernorat de Nabeul. M.E.A.T., Tunis; 70 p.

- Baccar, L., Moussa, M., et Ben Hamza, C., 2001. "Conservation des Zones Humides Littorales et des Ecosystèmes côtiers du Cap-Bon", in rapport L'hydraulique des zones humides de Maamoura Tazarka et Korba, http:/l www.apal.nat.tn/.../digitalAssets/194rapport_hydraulique.

- Benalaya, A., Chkirbene, A., Jallali, S., Harbaoui, K. and Tarhouni, J., 2009. «Evaluation de la qualité des eaux de la nappe de la côte orientale du Cap Bon en Tunisie ", article présenté dans le symposium international « Agriculture durable en région Méditerranéenne (AGDUMED), Rabat, $\quad$ Maroc. 2009. http://www.vulgarisation.net/agdumed2009/Benalaya. (Consulté le 7septembre 2012).

- Chaibi, T., Chenini, F., C. Epp, and G. Tondi. 2004. « Une approche intégrée pour la gestion durable des ressources en eau dans le bassin méditerranéen »http://www.francophoniedurable.org/.../colloque-ouaga-a3-chaibi (consulté le 28 aout 2012), p54

- Dervieux, A., 2005. « La difficile gestion globale de l'eau en Camargue (France): le Contrat de delta ",Vertigo - la revue électronique en sciences de l'environnement [En ligne], Volume 6 Numéro 3, consulté le 29 décembre 2012.

- URL: http://vertigo.revues.org/.p.2

- Gafsi, H., Ben Haj, S., 2009. Projet Destinations. Développement de stratégies pour un tourisme durable dans les nations méditerranéennes, Rapport diagnostic.p.18

- Ghariani,F,.2005. CAR/PAP: Gestion des zones côtières en Tunisie, Centre d'Activités Régionales pour le Programme d'Actions Prioritaires. Split, p.13, 16.

- Ghiotti, S., 2006. « Les Territoires de l'eau et la décentralisation. La gouvernance de bassin versant ou les limites d'une évidence », Développement durable et territoires, Dossier 6: Les territoires de l'eau, consulté le 14 décembre 2012.p.32

- Hammami.S., 2010, Territoires de projets et agriculture multifonctionnelle sur le littoral Tunisien, le cas de la façade orientale du Cap Bon, in mémoire de thèse de Hammami Saida. Institut Supérieur Agronomique de Chott-Meriem. Sousse, Tunisie.

- Khourey-Bowers Claudia, 2012. Éduquer à l'eau - Approches pluridisciplinaires. Éducation pour le développement durable en action. Outils d'apprentissage et de formation $N^{\circ} 5$ Document initial préparé pour l'UNESCO, Kent State University (USA)

- Makkaoui, R., et Dubois, JL., 2010 « Nouvelles formes de gouvernance dans le domaine de l'eau. Apports et limites de la coopération décentralisée dans les pays en développement », Développement durable et territoires [En ligne], Vol. 1, $n^{\circ} 1$, consulté le 01 janvier 2013. URL: http://developpementdurable.revues.org/8413.p. 23

- Mekki Hamza., 2006. État, défis majeurs et axes stratégiques du secteur de l'eau en Tunisie. In (eds.) Ministère de l'Agriculture et de l'Environnement, Tunis. 
- Mutin Georges., 2009. Le Monde arabe face au défi de l'eau. Enjeux et conflits. Institut d'Etudes Politiques de Lyon, p 3.

- OMERE., 2010. Le dispositif d'observation sur le bassin versant de Kamech, Cap Bon,

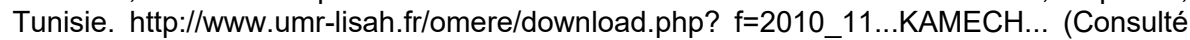
le 25 aout 2012)

- Piel. C., Veiga. L., Pire. M, 2010. Le cycle de l'eau, élément structurant d'une ville durable.

- Riviere-Honegger Anne, 2008. Regards sur les paysages de l'eau Evolution des usages de l'eau, dynamiques du territoire et mutations paysagères en Méditerranée occidentale. Rapport de synthèse d'Habilitation à Diriger des Recherches en géographie ENS-LSHLyon, volume 1, p 5.8 .65

- Romagny Bruno et Cudennec Christophe, 2006 «Gestion de l'eau en milieu aride: considérations physiques et sociales pour l'identification des territoires pertinents dans le Sud-Est tunisien », Développement durable et territoires [En ligne], Dossier 6: Les territoires de l'eau, mis en ligne le 10 février 2006, consulté le 03 janvier 2013. URL: http://developpementdurable.revues.org/1805, p.15-16

- Romdhane. MS; Missaoui. H; 2001. Conservation des Zones Humides Littorales et des Ecosystèmes côtiers du Cap-Bon. In Rapport de diagnostic des sites. Partie relative aux zones humides diagnostic hydrobiologique et étude des peuplements. Projet MedWetCoast. p.13

- Sethom. H., 1977. L'agriculture de la presqu'ile du Cap Bon (Tunisie) structures sociales et économie rurale. Publication de l'Université de Tunis, Tunisie, volume V, p.73-113-114.

- Temple-Boyer, E., 2006. «Aménagement et ménagement du paysage dans la dorsale Tunisienne, Evolution, représentation et gestion des eaux et des sols à l'échelle de trois petits bassins-versants ", Mémoire de thèse de doctorat, Ecole Normale Supérieure, Lettes et sciences humaines de Lyon.p.111-152.

- Treyer, S., 2000. Analyse des stratégies et prospectives de l'eau en Tunisie. Rapport consulté sur le site web: http://www.planbleu.org/publications/rapTreyer_eauTUN le 22 aout 2012. p. 16

- Zghibi, A., 2007. Evolution de l'état de l'aquifère plio-quaternaire de la côte orientale du Cap-Bon de la Tunisie (Etude hydrodynamique et Modélisation numérique) in mémoire de master en Hydraulique Agricole et Aménagement Rural. Institut National Agronomique de Tunis. http://www.memoireonline.com/04/08/1019/m_evolution-etat-aquifere-plioquaternaire-cap-bon-tunisie3.html, consulté le 30 aout 2012. 